\title{
Patterns of Surveillance Advanced Imaging and Serum Tumor Biomarker Testing Following Launch of the Choosing Wisely Initiative
}

Randy C. Miles, MD, MPHa; Christoph I. Lee, MD, MS ; ; Qin Sun, MPAc; Aasthaa Bansal, PhDd; Gary H. Lyman, MD, MPHe; Jennifer M. Specht, MDf; Catherine R. Fedorenko, MMScc; Mikael Anne Greenwood-Hickman, MPH';

Scott D. Ramsey, MD, PhDe; and Janie M. Lee, MD, MSc ${ }^{b}$

\section{ABSTRACT}

Background: The purpose of this study was to assess advanced imaging (bone scan, CT, or PET/CT) and serum tumor biomarker use in asymptomatic breast cancer survivors during the surveillance period. Patients and Methods: Cancer registry records for 2,923 women diagnosed with primary breast cancer in Washington State between January 1, 2007, and December 31, 2014, were linked with claims data from 2 regional commercial insurance plans. Clinical data including demographic and tumor characteristics were collected. Evaluation and management codes from claims data were used to determine advanced imaging and serum tumor biomarker testing during the peridiagnostic and surveillance phases of care. Multivariable logistic regression models were used to identify clinical factors and patterns of peridiagnostic imaging and biomarker testing associated with surveillance advanced imaging. Results: Of 2,923 eligible women, $16.5 \%(n=480)$ underwent surveillance advanced imaging and $31.8 \%(n=930)$ received surveillance serum tumor biomarker testing. Compared with women diagnosed before the launch of the Choosing Wisely campaign in 2012, later diagnosis was associated with lower use of surveillance advanced imaging (odds ratio [OR], $0.68 ; 95 \% \mathrm{Cl}, 0.52-0.89)$. Factors significantly associated with use of surveillance advanced imaging included increasing disease stage (stage III: OR, 3.65; 95\% Cl, 2.48-5.38), peridiagnostic advanced imaging use $(\mathrm{OR}, 1.76 ; 95 \% \mathrm{Cl}, 1.33-2.31)$, and peridiagnostic serum tumor biomarker testing (OR, 1.35; $95 \% \mathrm{Cl}, 1.01-1.80)$. Conclusions: Although use of surveillance advanced imaging in asymptomatic breast cancer survivors has declined since the launch of the Choosing Wisely campaign, frequent use of surveillance serum tumor biomarker testing remains prevalent, representing a potential target for further efforts to reduce low-value practices.

J Natl Compr Canc Netw 2019;17(7):813-820 doi: $10.6004 /$ jnccn.2018.7281

\footnotetext{
aDepartment of Radiology, Massachusetts General Hospital, Boston, Massachusetts; and 'bepartment of Radiology, University of Washington Medical Center, 'Fred Hutchinson Cancer Research Center, dUniversity of Washington, 'University of Washington Medical Center, 'Department of Oncology, University of Washington Medical Center, and 9 Kaiser Permanente Washington Health Research Institute, Seattle, Washington.
}

\section{Background}

The Institute of Medicine estimates that nearly $30 \%$ of all medical expenses result from inappropriate or unnecessary practices, increasing strain on the US healthcare system. ${ }^{1}$ In oncology, total healthcare expenditures are projected to reach $\$ 173$ billion by 2020 , a $\$ 50$ billion increase compared with $2010 .^{2}$ Efforts to identify and improve practices by physicians, payers, and policymakers led to the Choosing Wisely campaign by the ABIM Foundation (ABIMF).

In 2012, a "Top Five" list of low-value practices in oncology that could be avoided without impacting patient care was released through the Choosing Wisely initiative in partnership with ASCO., ${ }^{3,4}$ Surveillance testing of asymptomatic breast cancer survivors using advanced imaging (whole-body imaging with bone scan, CT, or PET/CT) and/ or serum tumor biomarkers (carcinoembryonic antigen, CA 15-3, and CA 27.29) was one measure included on this list. Randomized controlled trials have shown no survival benefit or decreased time to detection of disease recurrence in women receiving advanced imaging tests. ${ }^{5,6}$ Furthermore, numerous potential harms associated with advanced imaging use in breast cancer survivors have been reported, including increased patient anxiety, exposure to ionizing radiation, and false-positive results leading to further invasive testing.

Current NCCN and ASCO evidence-based guidelines for breast cancer follow-up and management after primary treatment include routine history, physical examination, and mammography. ${ }^{8,9}$ Physical examination is recommended every 3 to 6 months for the first 3 years, every 6 to 12 months for years 4 and 5 , and annually thereafter. For women who have received breastconserving therapy, a posttreatment mammogram is recommended 1 year after the initial mammogram and at least 6 months after completion of radiation therapy (RT), followed by yearly mammography.

Despite consensus on the Choosing Wisely recommendations advising against use of low-value surveillance 
care, variable adherence to these measures has been observed..$^{10}$ Predictors of nonconcordance with recommendations have yet to be clearly elucidated, which limits efforts to develop interventions aimed at reducing unnecessary surveillance testing. Understanding determinants of advanced surveillance testing will be key in implementing effective strategies to increase concordance with Choosing Wisely guidelines. Thus, the objectives of our study were to evaluate advanced imaging use during breast cancer surveillance and to identify drivers of potential advanced imaging overuse, as outlined by the ASCO/ABIMF Top Five recommendations.

\section{Patients and Methods}

A retrospective cohort study was performed involving 18,912 unique individuals diagnosed with breast cancer between January 1, 2007, and December 31, 2014. Insurance enrollment data from 2 major commercial insurers in the region, Premera Blue Cross and Regence Blue Shield, were linked with cancer registry records from the Cancer Surveillance System (CSS), which includes 13 counties in western Washington State, and claims for inpatient and outpatient services were extracted for eligible individuals during the study period. This HIPAA-compliant study received Institutional Review Board approval to link registry and commercial insurance data and to perform analyses.

We included women aged $\geq 18$ years at the time of diagnosis who had AJCC 7th edition stage 0 -III breast cancer who underwent primary treatment with curative intent (Figure 1). Primary therapy was defined as either mastectomy or lumpectomy with RT within 180 days of diagnosis. ${ }^{11}$ Exclusion criteria included women who (1) had a prior history of breast cancer (2) had an unknown breast cancer diagnosis date or a diagnosis date outside the study period, (3) were diagnosed at autopsy, (4) had AJCC stage IV breast cancer, (5) did not have continuous insurance enrollment from 3 months before diagnosis through 14 months after diagnosis, (6) did not receive curative therapy (ie, mastectomy 6 months after diagnosis or lumpectomy with RT within 180 days after surgery), and (7) did not have continuous insurance enrollment during the surveillance period (15-month period starting 4 months after completion of primary therapy).

Clinical data included age at diagnosis, race, diagnosis year, family history of breast cancer, and breast cancer-specific genetic mutation status. Tumor characteristic data included AJCC stage, grade, size, and estrogen receptor (ER), progesterone receptor (PR), and HER2 status. Survival information was obtained from the CSS.

Evaluation and management codes from claims data were used to determine advanced imaging (whole-body imaging with bone scan, CT, and/or PET/CT) and serum tumor biomarker testing during both the peridiagnostic period (ie, 1 month before diagnosis until completion of primary therapy) and the surveillance

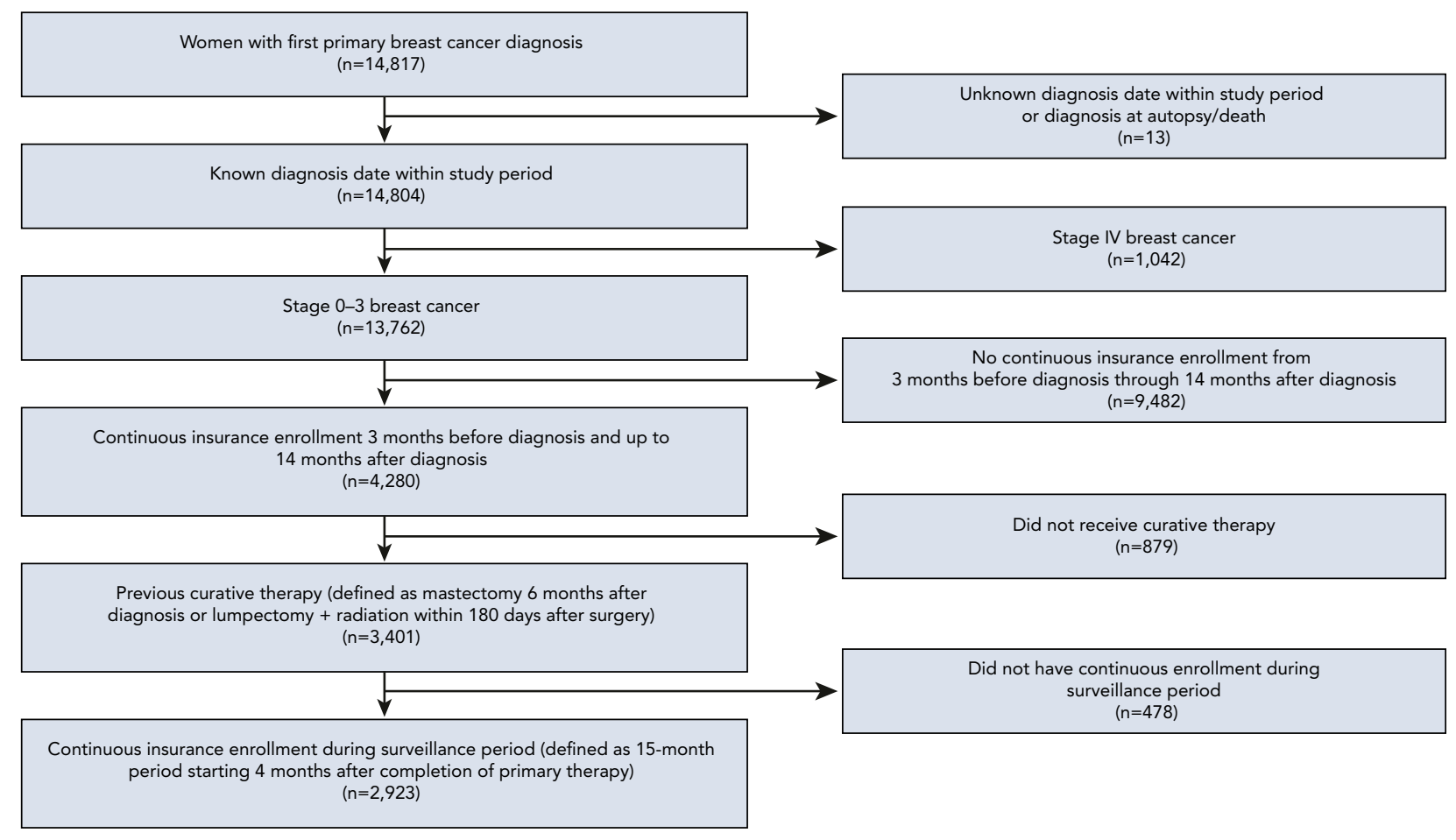

Figure 1. CONSORT diagram of cohort creation. 


\section{Table 1. Clinical and Tumor Characteristics of Study Cohort}

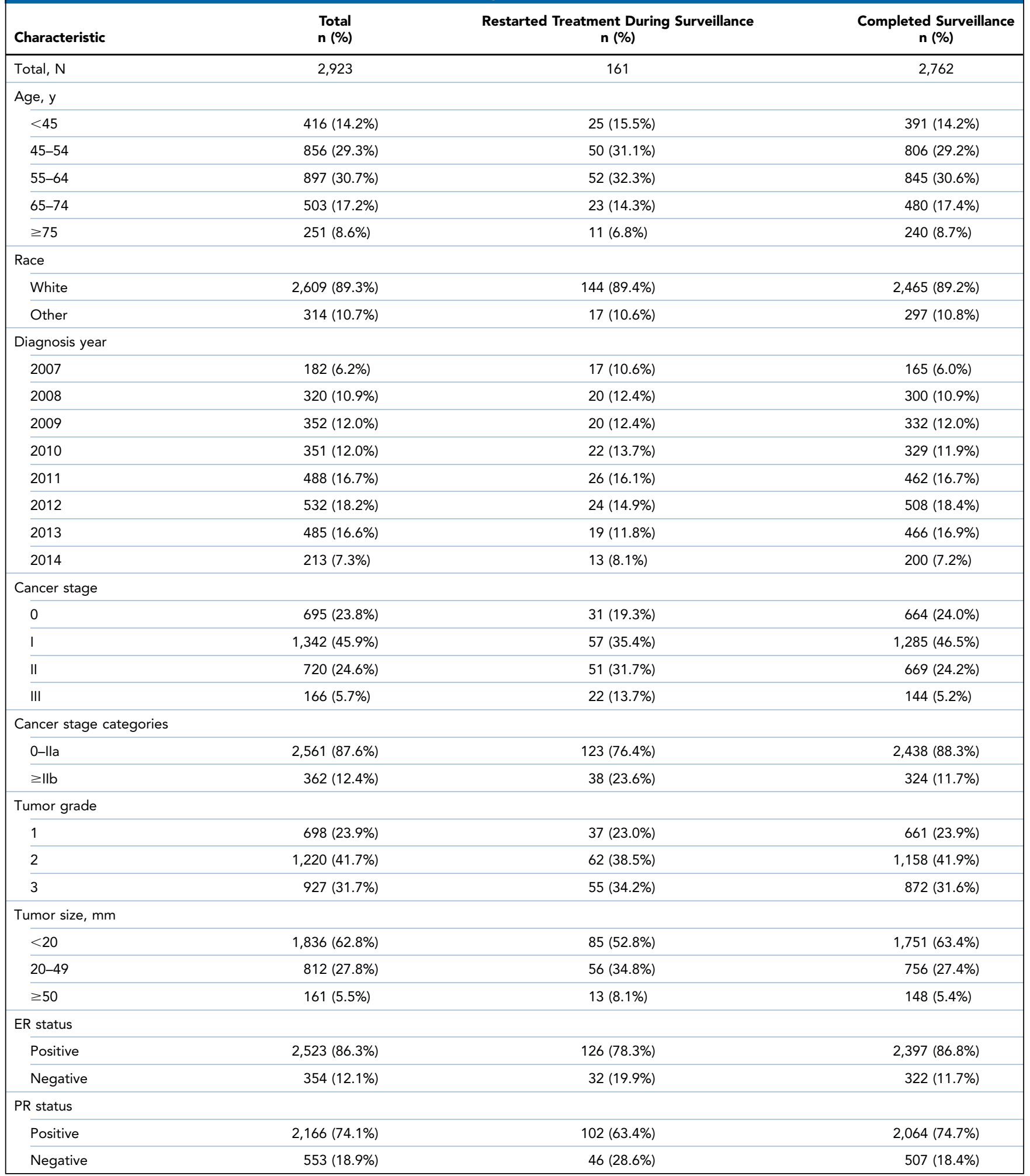

Abbreviations: ER, estrogen receptor; PR, progesterone receptor; $\mathrm{RT}$, radiation therapy. 


\begin{tabular}{|c|c|c|c|}
\hline Positive & 159 (5.4\%) & $10(6.2 \%)$ & 149 (5.4\%) \\
\hline Negative & $1,438(49.2 \%)$ & 71 (44.1\%) & 1,367 (49.5\%) \\
\hline Mastectomy & $1,131(38.7 \%)$ & 81 (50.3\%) & $1,050(38.0 \%)$ \\
\hline \multicolumn{4}{|l|}{ Family history } \\
\hline Yes & 641 (21.9\%) & 35 (21.7\%) & $606(21.9 \%)$ \\
\hline No & 2,282 (78.1\%) & 126 (78.3\%) & $2,156(78.1 \%)$ \\
\hline
\end{tabular}

Abbreviations: ER, estrogen receptor; PR, progesterone receptor; RT, radiation therapy.

period (ie, 15-month period starting 4 months after completion of primary therapy). ${ }^{12,13}$ Tumor biomarker testing performed after advanced imaging surveillance was excluded. Censoring occurred during surveillance at death or restart of treatment, defined as claims data documenting surgery, chemotherapy, or RT during surveillance, an end point that was used as a proxy for disease recurrence. ${ }^{10,14,15}$

Clinical and tumor characteristic data were compared between women who completed their surveillance period without further treatment and those who ended surveillance early due to restart of treatment. Use of advanced imaging and serum tumor biomarker testing was compared between women with less advanced versus more advanced disease (stage 0-IIa vs stage $\geq \mathrm{II}$, respectively) during both the peridiagnostic and surveillance periods. Use of surveillance advanced imaging and serum tumor biomarker testing in these 2 groups was then compared based on whether treatment was restarted during surveillance to assess potentially appropriate versus inappropriate use of surveillance testing.

Multivariable logistic regression was used to identify clinical factors and patterns of care (peridiagnostic advanced imaging and tumor biomarker testing and surveillance tumor biomarker testing) that were associated with surveillance advanced imaging. For the regression model, we excluded patients who underwent surveillance biomarker testing that did not precede surveillance advanced imaging. Specifically, the following covariates were included in the model: patient age and race; diagnosis year; cancer stage; ER, PR, and HER2 status; curative therapy; family history; genetic mutation; peridiagnostic advanced imaging; and peridiagnostic tumor biomarker testing. Patients with unknown ER/PR status were excluded. There was no interaction between covariates. All statistical tests were 2 -sided, and $P$ values $<.05$ were considered statistically significant.

For the fully adjusted multivariable model, we used multiple imputation via chained equations to impute

Table 2. Peridiagnostic Advanced Imaging and Serum Tumor Biomarker Testing

\begin{tabular}{|lccr|}
\hline & $\begin{array}{c}\text { All Women } \\
\mathbf{n}(\%)\end{array}$ & $\begin{array}{c}\text { Stage 0-Ila } \\
\mathbf{n}(\%)\end{array}$ & $\begin{array}{c}\text { Stage } \geq \text { Illb } \\
\mathbf{n}(\%)\end{array}$ \\
\hline Total, N & 2,923 & 2,561 & 362 \\
\hline No advanced imaging & $2,508(85.8 \%)$ & $2,306(90.0 \%)$ & $202(55.8 \%)$ \\
\hline Advanced imaginga & $415(14.2 \%)$ & $255(10.0 \%)$ & $160(44.2 \%)$ \\
\hline Bone scan & $134(4.6 \%)$ & $77(3.0 \%)$ & $57(15.7 \%)$ \\
\hline CT & $289(9.9 \%)$ & $182(7.1 \%)$ & $107(29.6 \%)$ \\
\hline PET/CT & $205(7.0 \%)$ & $101(3.9 \%)$ & $104(28.7 \%)$ \\
\hline Serum tumor biomarker testing & $388(13.3 \%)$ & $287(11.2 \%)$ & $101(27.9 \%)$ \\
\hline
\end{tabular}

alncluding any combination of bone scan, $\mathrm{CT}$, and/or PET/CT. 


\begin{tabular}{|c|c|c|c|}
\hline & $\begin{array}{c}\text { All Women } \\
\text { n (\%) }\end{array}$ & $\begin{array}{l}\text { Stage 0-Ila } \\
\text { n (\%) }\end{array}$ & $\begin{array}{c}\text { Stage } \geq \text { llb } \\
\text { n (\%) }\end{array}$ \\
\hline Total, N & 2,923 & 2,561 & 362 \\
\hline No advanced imaging & $2,443(83.5 \%)$ & 2,207 (86.2\%) & $236(65.2 \%)$ \\
\hline Advanced imaging ${ }^{a}$ & 480 (16.5\%) & 354 (13.8\%) & $126(34.8 \%)$ \\
\hline Bone scan & $132(4.5 \%)$ & $94(3.7 \%)$ & $38(10.5 \%)$ \\
\hline $\mathrm{CT}$ & 370 (12.7\%) & 266 (10.4\%) & $104(28.7 \%)$ \\
\hline $\mathrm{PET} / \mathrm{CT}$ & 85 (2.9\%) & $54(2.1 \%)$ & 31 (8.6\%) \\
\hline Serum tumor biomarker testing & 930 (31.8\%) & 748 (29.2\%) & $182(50.3 \%)$ \\
\hline
\end{tabular}

ancluding any combination of bone scan, CT, and/or PET/CT.

b185 women (6.3\%) received both surveillance advanced imaging and tumor biomarker use.

missing HER2 data in 1,123 women. ${ }^{16,17}$ This method imputed missing variables using a regression model conditional on all the other variables in the model. Ten imputations were conducted using STATA, version 14 (StataCorp LP), and estimates were combined across imputations using standard methods for multiple imputation. ${ }^{18}$

\section{Results}

After applying inclusion and exclusion criteria, 2,923 women were included in the final study cohort. Clinical data including demographic and tumor characteristics are shown in Table 1. Median age was 57.2 years (interquartile range, 48-65 years), and nearly half of all women ( $\mathrm{n}=1,342 ; 45.9 \%)$ were diagnosed with AJCC stage I disease. Most tumors were $<20 \mathrm{~mm}(\mathrm{n}=1,836 ; 62.8 \%)$, ER-positive ( $n=2,523 ; 86.3 \%)$, and PR-positive ( $n=2,166$; $74.1 \%)$. Most women $(n=1,792 ; 61.3 \%)$ underwent breast conservation therapy, whereas $38.7 \%(n=1,131)$ underwent mastectomy. A minority of women had a family history of breast cancer $(n=641 ; 21.9 \%)$ or breast cancerspecific genetic mutation $(n=52 ; 1.8 \%)$. The surveillance period ended early for $5.5 \%$ of women $(n=161)$ due to restart of treatment; these women were more likely to have advanced disease stage, larger tumor size $(>20 \mathrm{~mm})$, and ER-negative status at primary breast cancer diagnosis compared with those who did not restart treatment.

During the peridiagnostic phase of care, $14.2 \%$ of all women $(n=415)$ received at least 1 advanced imaging test (bone scan, CT, and/or PET/CT) and $13.3 \%(n=388)$ received serum tumor biomarker testing (Table 2). Additionally, compared with those with early-stage breast cancer (stage 0 -IIa; $n=2,561$ ), a greater proportion with advanced-stage breast cancer (stage $\geq \mathrm{IIb}$; $\mathrm{n}=362$ ) received advanced imaging ( $44.2 \%$ vs $10.0 \%)$ and serum tumor biomarker testing (27.9\% vs $11.2 \%)$.

During the surveillance phase of care, $16.5 \%$ of all women $(n=480)$ received advanced imaging and $31.8 \%$ $(n=930)$ received serum tumor biomarker testing (Table 3$)$.
CT scan $(n=370)$ was the most frequently used advanced imaging modality, followed by bone scan $(n=132)$ and PET/CT $(n=85)$. Compared with those with early-stage breast cancer, more women with advanced-stage breast cancer received advanced imaging surveillance $(34.8 \%$ vs $13.8 \%$ ) and serum tumor biomarker testing (50.3\% vs $29.2 \%$ ). Serum tumor biomarker testing among all women increased from $13.3 \%(388 / 2,923)$ to $31.8 \%$ $(930 / 2,923)$ between the peridiagnostic and surveillance phases of care.

Multivariable logistic regression modeling indicated that advanced imaging use during surveillance was most significantly associated with increasing disease stage (stage III: OR, 3.65; 95\% CI, 2.48-5.38) (Table 4). Compared with women diagnosed before the launch of Choosing Wisely in 2012, later diagnosis was associated with lower use of advanced imaging surveillance (OR, 0.68; 95\% CI, $0.52-0.89$ ). Additional factors significantly associated with advanced imaging surveillance included peridiagnostic advanced imaging (OR, 1.76; 95\% CI, 1.33-2.31) and serum tumor biomarker testing (OR, 1.35; 95\% CI, 1.01-1.80).

Among women who received advanced imaging during surveillance $(n=480), 19.1 \%(24 / 126)$ with advancedstage breast cancer restarted treatment compared with $6.5 \%(23 / 354)$ with early-stage breast cancer (Table 5). More than $90 \%$ of women with early-stage breast cancer who received either advanced imaging or serum tumor biomarker testing during the surveillance phase of care did not restart treatment.

\section{Discussion}

Several studies have shown limited clinical benefit from advanced imaging surveillance and serum tumor biomarker testing in asymptomatic breast cancer survivors. ${ }^{5-7}$ In our study population of 2,923 women treated for primary breast cancer with curative intent, we found that only $16.5 \%$ received surveillance advanced imaging, representing a lower proportion compared with prior studies evaluating advanced imaging use among breast cancer 


\section{Table 4. Multivariable Analysis of Association Between Clinical Characteristics and Surveillance Advanced Imaging}

\begin{tabular}{|c|c|c|}
\hline Variable ${ }^{a}$ & Odds Ratio $(95 \% \mathrm{Cl})$ & $P$ Value \\
\hline Age, y & & .41 \\
\hline$<45$ & $1.18(0.84-1.67)$ & \\
\hline $45-54$ & $1.27(0.96-1.67)$ & \\
\hline $55-64$ & Ref & \\
\hline $65-74$ & $1.18(0.85-1.63)$ & \\
\hline$\geq 75$ & $1.43(0.97-2.11)$ & \\
\hline Race & & .93 \\
\hline White & Ref & \\
\hline Nonwhite & $1.02(0.71-1.46)$ & \\
\hline Diagnosis year & & $<.01$ \\
\hline $2007-2012$ & Ref & \\
\hline 2013-2014 & $0.68(0.52-0.89)$ & \\
\hline Cancer stage & & $<.01$ \\
\hline 0 & $0.55(0.38-0.80)$ & \\
\hline 1 & Ref & \\
\hline II & $1.85(1.45-2.37)$ & \\
\hline III & $3.65(2.48,5.38)$ & \\
\hline ER status & & .33 \\
\hline Positive & $0.82(0.54-1.23)$ & \\
\hline Negative & Ref & \\
\hline PR status & & .36 \\
\hline Positive & $0.85(0.60-1.20)$ & \\
\hline Negative & Ref & \\
\hline HER2 status & & .96 \\
\hline Positive & $0.99(0.64-1.52)$ & \\
\hline Negative & Ref & \\
\hline Curative therapy & & .86 \\
\hline Mastectomy & Ref & \\
\hline Lumpectomy + RT & $0.98(0.78-1.23)$ & \\
\hline Family history & & .63 \\
\hline Yes & $1.07(0.82-1.38)$ & \\
\hline No & Ref & \\
\hline Genetic mutation & & .46 \\
\hline Yes & $1.30(0.65-2.60)$ & \\
\hline No & Ref & \\
\hline Tumor marker testing (peridiagnostic) & & .04 \\
\hline Yes & $1.35(1.01-1.80)$ & \\
\hline No & Ref & \\
\hline
\end{tabular}

Abbreviations: $E R$, estrogen receptor; $\mathrm{PR}$, progesterone receptor; $\mathrm{RT}$ (continued) radiation therapy.

${ }^{a}$ Covariates included in the model: patient age and race; diagnosis year; cancer stage; ER, PR, and HER2 status; curative therapy; family history; genetic mutation; peridiagnostic advanced imaging; and peridiagnostic tumor biomarker testing.

\section{Table 4. Multivariable Analysis of Association} Between Clinical Characteristics and Surveillance Advanced Imaging (cont.)

\section{Variable $^{\mathbf{a}}$}

Odds Ratio $(95 \% \mathrm{Cl}) \quad$ P Value

Tumor marker testing (surveillance)

$$
\text { Yes }
$$

$$
\text { No }
$$

Advanced imaging use (peridiagnostic)

$$
\text { Yes }
$$

No

$0.86(0)$

$0.86(0.68-1.08)$

Ref

Abbreviations: ER, estrogen receptor; PR, progesterone receptor; RT, radiation therapy.

${ }^{a}$ Covariates included in the model: patient age and race; diagnosis year; cancer stage; ER, PR, and HER2 status; curative therapy; family history; genetic mutation; peridiagnostic advanced imaging; and peridiagnostic tumor biomarker testing

survivors. ${ }^{10}$ Nearly one-third of women in this study received surveillance serum tumor biomarker testing, however, reflecting that care that may be discordant with Choosing Wisely guideline recommendations. ${ }^{3,4}$

Of women receiving surveillance advanced imaging, $6.5 \%(23 / 354)$ treated for early-stage disease and $19.1 \%$ $(24 / 126)$ treated for advanced-stage disease restarted treatment because of probable recurrent or metastatic disease. In contrast, overall low treatment restart rates were seen in women receiving surveillance serum tumor biomarker testing $(5.3 \%$; $49 / 930)$ for probable recurrent or metastatic disease, despite nearly one-third of survivors of primary breast cancer receiving these tests. Additionally, increased surveillance tumor biomarker testing was not associated with follow-up surveillance advanced imaging, indicating that biomarker testing had little effect on subsequent clinical management and supporting its limited clinical benefit in this population.

Overall, variable surveillance advanced imaging estimates ranging from $24 \%$ to $55 \%$ have been previously reported, likely attributable to differences in study setting, patient selection, and definitions of advanced imaging surveillance. ${ }^{19-23}$ Panageas et al, ${ }^{23}$ for example, who showed advanced imaging surveillance use in $40 \%$ of early-stage breast cancer survivors using SEERMedicare linked data, included breast MRI in their analysis, likely contributing to comparatively higher overall advanced imaging use rates. Our study results were more aligned with more recent studies showing lower use rates, supporting decreasing use of surveillance advanced imaging over time. ${ }^{9,20}$ In a recent analysis, Schumacher et $\mathrm{al}^{20}$ found that although patients were more likely to receive surveillance imaging at their first follow-up examination, lower use was seen in subsequent years. Similar to our findings, their study supported surveillance imaging use in patients with 


\begin{tabular}{|c|c|c|c|}
\hline & \multicolumn{2}{|c|}{ Treatment Restarted } & \multirow[b]{2}{*}{$\begin{array}{c}\text { Total, } \\
\mathbf{N}\end{array}$} \\
\hline & $\begin{array}{c}\text { Yes }^{\mathrm{a}} \\
\text { n (\%) }\end{array}$ & $\begin{array}{c}\text { No } \\
\text { n (\%) }\end{array}$ & \\
\hline \multicolumn{4}{|c|}{ Advanced imaging performed } \\
\hline Stage $0-11$ a & $23(6.5 \%)$ & 331 (93.5\%) & 354 \\
\hline Stage $\geq l l b$ & 24 (19.1\%) & 102 (80.9\%) & 126 \\
\hline Total & 47 (9.8\%) & 433 (90.2\%) & 480 \\
\hline \multicolumn{4}{|c|}{ Serum tumor biomarker performed } \\
\hline Stage $0-1 l a$ & $33(4.4 \%)$ & 715 (95.6\%) & 748 \\
\hline Stage $\geq 11 b$ & $16(8.8 \%)$ & $166(91.2 \%)$ & 182 \\
\hline Total & 49 (5.3\%) & 881 (94.7\%) & 930 \\
\hline
\end{tabular}

aPotentially appropriate use of surveillance testing for recurrence based on claims data documenting surgery, chemotherapy, or radiotherapy during surveillance.

higher recurrence risk, including those with ER/PRnegative tumors. Greater awareness of the Choosing Wisely initiative may influence this trend toward more evidence-based surveillance practice patterns that has been reported in more recent studies, ${ }^{24}$ as well as expansion of high-deductible health plans in the private market over time. ${ }^{25}$ This possibility is supported by our findings, which showed that earlier date of primary breast cancer diagnosis, before the launch of the Choosing Wisely campaign in 2012, was a predictor of surveillance advanced imaging use. Lastly, prior authorization requirements for surveillance testing by health insurance companies for women with early-stage breast cancer may have influenced study results, although not directly evaluated in our study. ${ }^{26}$

Advanced imaging and tumor biomarker testing performed during the peridiagnostic phase of care was associated with increased use of surveillance advanced imaging. Considering that these tests were performed before surgery, it seems that peridiagnostic ordering patterns may significantly influence surveillance advanced imaging use by potentially informing recurrence risk. These findings may be supported by recent data showing a positive association between surveillance PET imaging use and mastectomy $+\mathrm{RT}$, indicating that rates of advanced imaging use may be higher in women with increased risk of recurrence. ${ }^{27}$ Patient age, family history, and tumor characteristics represent additional factors that may influence perception of recurrence risk in breast cancer survivors. ${ }^{15,19}$ Of these previously studied factors, however, only increasing disease stage was associated with surveillance advanced imaging use in our study.

Variation in regional practice patterns and guideline adherence may also influence clinical decision-making when considering surveillance imaging. ${ }^{28}$ Although oncologists have been shown to make guideline-concordant decisions in most clinical scenarios, ${ }^{29}$ higher instances of guideline-discordant care have been reported regarding the diagnostic use of PET imaging and serum tumor biomarker tests in early-stage breast cancer survivors, ${ }^{15,21,29}$ similar to our findings. Although clinician unfamiliarity with Choosing Wisely guidelines may play a partial role, anecdotal experience with perceived benefits of nonrecommended tests and reliance on smaller studies may also contribute to guideline-discordant surveillance care.

Our study has several limitations. Claims data do not include information on clinical indication or test results, which limits assessment of the appropriateness of followup testing based on suspicious clinical history and/or physical examination findings. Additionally, limited data were available on the number of patients per physician and clinic with which to analyze physician- and clinic-level variables. Detailed information on preauthorization policies and pathways was also limited in our dataset. ${ }^{15}$ Lastly, because our study focused on women receiving guideline-concordant surgical therapy, we did not include those receiving lumpectomy without RT, who are at greater risk of local recurrence. As a result, our study results may not be generalizable to this small subpopulation of women.

\section{Conclusions}

As breast cancer survivorship continues to increase, the potential negative impact of inappropriate surveillance testing on individuals, and the US healthcare system overall, will only be magnified. ${ }^{30,31}$ Prior reports demonstrating potential imaging overuse highlight the importance of evidence-based initiatives such as the Choosing Wisely campaign. ${ }^{32}$ Moreover, it is unclear how recently established guidelines by the American College of Radiology suggesting annual breast MRI in breast cancer survivors aged $>50$ years or who have dense breast tissue will impact messaging around appropriate use of advanced imaging in this population. ${ }^{33}$ Although our study shows that guideline-discordant care may be declining after the launch of Choosing Wisely, nonrecommended surveillance testing remains prevalent. Continued efforts to reduce low-value care are needed to reduce costly, unnecessary tests and procedures, with special focus on surveillance tumor biomarker testing.

Initiatives such as ASCO's Quality Oncology Practice Initiative, an oncologist-led, practice-based quality assessment and improvement program, aim to promote evidence-based cancer care by encouraging constant self-inspection to reduce discordant practice patterns while creating a platform to compare variation across diverse practices. ${ }^{34}$ Peridiagnostic ordering patterns that may lead to guideline-discordant surveillance testing, as seen in our study, could be documented, compared, and used to target physicians who are more likely to not adhere to Choosing Wisely guidelines. Overall, increased efforts to educate health practitioners and patients regarding 
evidence-based breast cancer surveillance practices will be required to further increase concordance with Choosing Wisely guidelines.

Submitted September 5, 2018; accepted for publication February 6, 2019.

Author contributions: Data acquisition: Miles, Sun, Bansal, Fedorenko, Greenwood-Hickman. Data analysis: Miles, C.I. Lee, Sun, Bansal, Fedorenko,
Greenwood-Hickman. Manuscript preparation: Miles, C.I. Lee, Lyman, Specht, Ramsey, J.M. Lee.

Disclosures: Dr. J.M. Lee has disclosed that she receives grant/research support from GE Healthcare. The remaining authors have disclosed that they have not received any financial considerations from any person or organization to support the preparation, analysis, results, or discussion of this article.

Correspondence: Randy C. Miles, MD, MPH, Department of Radiology, Massachusetts General Hospital, 55 Fruit Street, Boston, MA 02141. Email: randy.miles@mgh.harvard.edu

\section{References}

1. Institute of Medicine. Best Care at Lower Cost: The Path to Continuously Learning Health Care in America. Washington, DC: The National Academies Press; 2013.

2. Mariotto $A B$, Yabroff $K R$, Shao $Y$, et al. Projections of the cost of cancer care in the United States: 2010-2020. J Natl Cancer Inst 2011;103:117-128.

3. Schnipper LE, Lyman GH, Blayney DW, et al. American Society of Clinical Oncology 2013 top five list in oncology. J Clin Oncol 2013;31:4362-4370.

4. Schnipper LE, Smith TJ, Raghavan D, et al. American Society of Clinical Oncology identifies five key opportunities to improve care and reduce costs: the top five list for oncology. J Clin Oncol 2012;30:1715-1724.

5. Liberati A. The GIVIO trial on the impact of follow-up care on survival and quality of life in breast cancer patients. Ann Oncol 1995;6(Suppl 2):41-46.

6. Rosselli Del Turco M, Palli D, Cariddi A, et al. Intensive diagnostic followup after treatment of primary breast cancer. A randomized trial. JAMA 1994;271:1593-1597.

7. Jochelson M, Hayes DF, Ganz PA. Surveillance and monitoring in breast cancer survivors: maximizing benefit and minimizing harm. Am Soc Clin Oncol Educ Book 2013;33:e13-18.

8. Gradishar WJ, Anderson BO, Abraham J, et al. NCCN Clinical Practice Guidelines in Oncology: Breast Cancer. Version 1.2019. Accessed May 18 2019. To view the most recent version, visit NCCN.org

9. Khatcheressian JL, Hurley P, Bantug E, et al. Breast cancer follow-up and management after primary treatment: American Society of Clinical Oncology clinical practice guideline update. J Clin Oncol 2013;31:961-965

10. Ramsey SD, Fedorenko C, Chauhan R, et al. Baseline estimates of adherence to American Society of Clinical Oncology/American Board of Internal Medicine Choosing Wisely initiative among patients with cancer enrolled with a large regional commercial health insurer. J Oncol Pract 2015;11:338-343

11. Edge SB, Compton CC. The American Joint Committee on Cancer: the 7 th edition of the AJCC cancer staging manual and the future of TNM. Ann Surg Oncol 2010;17:1471-1474.

12. Stout NK, Nekhlyudov L, Li L, et al. Rapid increase in breast magnetic resonance imaging use: trends from 2000 to 2011. JAMA Intern Med 2014;174:114-121.

13. Chubak J, Yu O, Pocobelli G, et al. Administrative data algorithms to identify second breast cancer events following early-stage invasive breast cancer. J Natl Cancer Inst 2012;104:931-940.

14. Hassett MJ, Ritzwoller DP, Taback N, et al. Validating billing/encounter codes as indicators of lung, colorectal, breast, and prostate cancer recurrence using 2 large contemporary cohorts. Med Care 2014;52:e65-73.

15. Loggers ET, Buist DS, Gold LS, et al. Advanced imaging and receipt of guideline concordant care in women with early stage breast cancer. Int J Breast Cancer 2016;2016:2182985.

16. Raghunathan TW, Lepkowski JM, Van Hoewyk J, et al. A multivariate technique for multiply imputing missing values using a sequence of regression models. Surv Methodol 2001;27:85-95.

17. van Buuren S. Multiple imputation of discrete and continuous data by fully conditional specification. Stat Methods Med Res 2007;16:219-242.
18. Little RJ, Rubin DB. Statistical Analysis With Missing Data. New York, NY J. Wiley \& Sons; 1987

19. Hahn EE, Tang T, Lee JS, et al. Use of posttreatment imaging and biomarkers in survivors of early-stage breast cancer: inappropriate surveillance or necessary care? Cancer 2016;122:908-916.

20. Schumacher JR, Neuman HB, Chang GJ, et al. A national study of the use of asymptomatic systemic imaging for surveillance following breast cancer treatment (AFT-01). Ann Surg Oncol 2018;25:2587-2595.

21. Hahn EE, Hays RD, Kahn KL, et al. Use of imaging and biomarker tests for posttreatment care of early-stage breast cancer survivors. Cancer 2013 ; 119:4316-4324

22. Grunfeld E, Hodgson DC, Del Giudice ME, et al. Population-based longitudinal study of follow-up care for breast cancer survivors. J Oncol Pract 2010;6:174-181

23. Panageas KS, Sima CS, Liberman L, et al. Use of high technology imaging for surveillance of early stage breast cancer. Breast Cancer Res Treat 2012; 131:663-670.

24. Horný M, Burgess JF Jr, Cohen AB. Advanced imaging utilization trends in privately insured patients from 2007 to 2013. J Am Coll Radiol 2015; 12(12 Pt B):1380-1387.e4.

25. Public Law 109-176_Feb 8, 2006. Deficit Reduction Act of 2005 Available at: https://www.govinfo.gov/content/pkg/PLAW-109publ171/ pdf/PLAW-109publ171.pdf. Accessed July 15, 2018.

26. Henry NL, Braun TM, Breslin TM, et al. Variation in the use of advanced imaging at the time of breast cancer diagnosis in a statewide registry. Cancer 2017;123:2975-2983.

27. Franc BL, Copeland TP, Thombley R, et al. Geographic variation in postoperative imaging for low-risk breast cancer. J Natl Compr Canc Netw 2018;16:829-837.

28. Makarov DV, Soulos PR, Gold HT, et al. Regional-level correlations in inappropriate imaging rates for prostate and breast cancers: potential implications for the Choosing Wisely campaign. JAMA Oncol 2015;1: 185-194.

29. Foster JA, Abdolrasulnia M, Doroodchi $H$, et al. Practice patterns and guideline adherence of medical oncologists in managing patients with early breast cancer. J Natl Compr Canc Netw 2009;7:697-706.

30. Miller KD, Siegel RL, Lin CC, et al. Cancer treatment and survivorship statistics, 2016. CA Cancer J Clin 2016;66:271-289.

31. Baxi SS, Kale M, Keyhani S, et al. Overuse of health care services in the management of cancer: a systematic review. Med Care 2017;55:723-733.

32. Medicare Payment Advisory Commission. Report to the Congress: Medicare and the Health Care Delivery System. Available at: http:// medpac.gov/docs/default-source/reports/jun18_medpacreporttocongress_ sec.pdf. Accessed May 21, 2019.

33. Monticciolo DL, Newell MS, Moy L, et al. Breast cancer screening in women at higher-than-average risk: recommendations from the ACR. J Am Coll Radiol 2018;15:408-414.

34. Rocque $G$, Blayney DW, Jahanzeb $M$, et al. Choosing wisely in oncology: are we ready for value-based care? J Oncol Pract 2017;13:e935-943. 